\title{
Characterization of a Riboflavin Non-aqueous Nanosuspension Prepared by Bead Milling for Cutaneous Application
}

\author{
Takayuki Sato, ${ }^{* a, b}$ Hiroyuki Takeuchi, ${ }^{b}$ Takanobu Sakurai, ${ }^{c}$ Kensuke Tanaka, ${ }^{b}$ Kota Matsuki, ${ }^{b}$ \\ Kenjirou Higashi, ${ }^{a}$ Kunikazu Moribe, ${ }^{a}$ and Keiji Yamamoto ${ }^{a}$ \\ ${ }^{a}$ Graduate School of Pharmaceutical Sciences, Chiba University; 1-8-1 Inohana, Chuo-ku, Chiba 260-8675, Japan: \\ ${ }^{b}$ Pharmaceutics, Pharmaceutical Science Laboratories, Research Headquarters, Taisho Pharmaceutical Co., Ltd.; \\ 1-403 Yoshino-cho, Kita-ku, Saitama 331-9530, Japan: and ${ }^{c}$ Drug Safety, Drug Safety and Pharmacokinetics \\ Laboratories, Research Headquarters, Taisho Pharmaceutical Co., Ltd.; 1-403 Yoshino-cho, Kita-ku, Saitama 331- \\ 9530, Japan. \\ Received September 7, 2014; accepted November 11, 2014
}

\begin{abstract}
The purpose of this study was to characterize the non-aqueous nanosuspension of a hydrophilic drug prepared by bead milling for cutaneous application. Riboflavin was used as the model hydrophilic drug. The non-aqueous nanosuspensions were prepared by grinding riboflavin with zirconia beads using eight nonaqueous bases. The mean particle size of riboflavin in the suspensions ranged from 206 to $469 \mathrm{~nm}$, as determined by the dynamic light scattering method. Among the well-dispersed samples, riboflavin nanosuspension prepared in oleic acid was selected for evaluation of the drug permeability through rat skin. The cumulative amount and permeation rate of riboflavin from the nanosuspension were approximately three times higher than those for unprocessed riboflavin in oleic acid. Fluorescence imaging of the riboflavin nanosuspension suggested improved penetration of riboflavin into the stratum corneum. Furthermore, the addition of polysorbate 65 or polyglyceryl-6 polyricinoleate to the nanosuspension prepared in oleic acid markedly improved the riboflavin dispersibility. These results show that the preparation of a nanosuspension in a non-aqueous base by bead milling is one of the simple methods to improve the skin permeability of hydrophilic drugs.
\end{abstract}

Key words bead milling; riboflavin; non-aqueous nanosuspension; oleic acid; stabilization; skin permeability

The skin permeability of hydrophilic drugs is generally known to be low, ${ }^{1,2)}$ due to the existence of lipophilic stratum corneum. It is difficult to obtain sufficient skin permeation to obtain a pharmacological effect without skin irritation, even with the use of chemical enhancers. ${ }^{3)}$ Application of hydrophilic drugs by iontophoresis ${ }^{4,5)}$ and microneedle, ${ }^{6,7)}$ known as physical enhancers, allows dramatic improvement of the skin permeation, but these devices are expensive, and the safety of their long-term use still needs to be evaluated.

In recent years, application of the preparations for cutaneous with the use of nanoparticles has been reported. Polymer nanoparticles composed of poly(DL-lactic-co-glycolic acid) ${ }^{8,9)}$ or chitosan ${ }^{9,10)}$ are considered to enhance the skin permeation of drugs by improving the polymer penetration into hair follicles. Solid-in-oil $(\mathrm{S} / \mathrm{O})$ nanosuspension is reported to enhance the skin permeation of hydrophilic drugs via penetration of the nanoparticles into the stratum corneum, ${ }^{11)}$ and penetration into the stratum corneum of latex-beads nanoparticles has been confirmed. ${ }^{12)}$ In addition, solid-lipid nanoparticles and nanostructured lipid carriers enhance drug permeation by forming a film on the skin, improving the skin hydration. ${ }^{13)}$ However, the presence of nanoparticles in the receptor fluid was not shown in these reports.

Nanosuspensions are bothersome to produce via as the process involves emulsification, drying/evaporation/centrifugation, and redispersion. Especially, preparation of uniformly dispersed nanosuspensions is difficult because of the agglomeration forces between nanoparticles after the drying/evaporation/centrifugation process. In addition, nanosuspensions are suitable for sustained-release, ${ }^{14,15}$ not for immediate-release, because the drug is contained within the polymer or lipid matrix.

For reducing the sizes of drug crystals, the wet milling technology using beads has attracted attention. The technology has been used to prepare nanosuspensions of poorly water-soluble drugs for oral formulations. Bead milling enables preparation of nanoparticles only by mixing the components: drug, surfactant, and polymer with beads in water. ${ }^{16-18)}$ Size reduction using bead milling has not only the advantage of allowing preparation of nanosuspensions at high concentrations, but can also be scaled up. In addition, it is not necessary to perform the redispersion process, which is one of the most serious problems in the preparation of nanosuspensions.

Riboflavin, the name given to vitamin $\mathrm{B}_{2}$, is a hydrophilic compound. The skin permeability of riboflavin is predicted to be low, with a $\log K_{\mathrm{o} / \mathrm{w}}$ value of $-1.46 .{ }^{19)}$ Riboflavin is efficacious against pellagra, acne vulgaris and solar dermatitis, which caused by riboflavin deficiency or metabolic disorders. Oral dosage forms and injectable forms of riboflavin have been launched in the market as ethical drugs. Although preparations for cutaneous application have not been released, a topical cosmetic cream containing riboflavin with other $\mathrm{B}$ vitamins is only on the market. ${ }^{20)}$

The purpose of this study is to evaluate nanonization effect of the hydrophilic drug on the skin permeation, which becomes one of the basic data to develop the non-aqueous formulation. Bead milling technique was applied to prepare a nanosuspension of riboflavin, a model hydrophilic drug, in a non-aqueous base for cutaneous application. Bead milling was performed by grinding with zirconia beads in a nonaqueous base. Eight non-aqueous bases (liquid paraffin, isopropyl myristate, propylene glycol dicaprylate, caprylic/capric 
triglyceride, oleic acid, oleyl alcohol, triacetin, and propylene carbonate), which are the most commonly used for preparations for cutaneous application, were selected. For these suspensions prepared by the bead milling technique, the particle size, dispersion ratio and skin permeability were evaluated. Furthermore, from the point of view of practical application, the dispersion stabilization of the nanosuspension was investigated by adding several surfactants.

\section{Experimental}

Materials Riboflavin, with a particle size of $75-150 \mu \mathrm{m}$, which was sieved using Riboflavin High Flow 100 acquired from BASF Japan Ltd. (Tokyo, Japan), was used. Oleic acid (OC) and propylene carbonate (PC) were purchased from Wako Pure Chemical Industries, Ltd. (Osaka, Japan). Oleyl alcohol (OL) and triacetin (TA) were obtained from SigmaAldrich Co., LLC. (MO, U.S.A.). Isopropyl myristate (IM), propylene glycol dicaprylate (PD), caprylic/capric triglyceride (CT), sorbitan trioleate (ST), polysorbate 65 (PS), and polyglyceryl-6 polyricinoleate (PP) were procured from Nikko Chemicals Co., Ltd. (Tokyo, Japan). Liquid paraffin (LP) and sucrose erucate (SE) were purchased from Kaneda Co., Ltd. (Tokyo, Japan) and Mitsubishi-Kagaku Foods Corporation (Tokyo, Japan), respectively. All other chemicals and solvents were of reagent grade.

Viscosity of the Non-aqueous Bases The viscosities of the non-aqueous bases were determined by the vibration method. Data were obtained using VM-100 A (Yamaichi Electronics Co., Ltd., Tokyo, Japan). The viscosity value was fixed after the probe was immersed for $1 \mathrm{~min}$.

Density of the Non-aqueous Bases The densities of the non-aqueous bases were determined by measuring the mass of $10 \mathrm{~mL}$ of the non-aqueous base using a volumetric flask at $25 \pm 2{ }^{\circ} \mathrm{C}$.

Riboflavin Solubility in the Non-aqueous Bases Thirty milligram of riboflavin and $2.97 \mathrm{~g}$ of the non-aqueous base were added into a glass tube and shaken for $24 \mathrm{~h}$ at $25 \pm 2{ }^{\circ} \mathrm{C}$. After filtering through a $0.45-\mu \mathrm{m}$ filter, the filtrate was centrifuged for $10 \mathrm{~min}$ at $15000 \mathrm{rpm}$. After the supernatant was collected, the sample solution was obtained by 40 -fold dilution with tetrahydrofuran. Five microliters of the sample solution was injected to HPLC to determine the solubility of riboflavin.

Bead Milling Any of nanosuspensions were prepared as follows. Two hundred fifty milligram of riboflavin, $24.8 \mathrm{~g}$ of the non-aqueous base, and $150 \mathrm{~g}$ of zirconia beads (diameter: $0.1 \mathrm{~mm}, 0.5 \mathrm{~mm}, 1.0 \mathrm{~mm}$ ) were placed in a $200-\mathrm{mL}$ glass beaker and stirred for $15 \mathrm{~min}$ to $6 \mathrm{~h}$ at $1000 \mathrm{rpm}$ using a stirrer (Yamato Scientific Co., Ltd., Tokyo, Japan) under dark condition. The riboflavin nanosuspensions (NSs) were prepared using LP, IM, PD, CT, OC, OL, TA, and PC.

Powder X-Ray Diffraction Measurement Powder X-ray diffraction patterns were determined using RINT2200 U1timaIII (Rigaku, Tokyo, Japan) under the following operating conditions: target, copper; filter, nickel; voltage, $40 \mathrm{kV}$; current, $40 \mathrm{~mA}$; scanning speed, $4 \% \mathrm{~min}$; scanning angle, $3-40^{\circ}$. Five milliliter of the nanosuspension was centrifuged for $10 \mathrm{~min}$ at $3000 \mathrm{rpm}$. Then, $1.5 \mathrm{~mL}$ of the precipitate was centrifuged for $10 \mathrm{~min}$ at $15000 \mathrm{rpm}$. After adding $1 \mathrm{~mL}$ of hexane to this residue, centrifuging the solution for $10 \mathrm{~min}$ at $15000 \mathrm{rpm}$, and drying at room temperature, the measurement sample was obtained.
Particle Size Measurement The patterns of the particle size distribution as Gaussian were determined by the dynamic light scattering method. The mean volume-weighted particle sizes of riboflavin were evaluated using Nicomp 380 ZLS (Particle Sizing Systems, FL, U.S.A.) equipped with a $\mathrm{He}-\mathrm{Ne}$ laser at $632.8 \mathrm{~nm}$. The suspensions prepared in LP, IM, PD, CT, OC, and OL were diluted 100 -fold with $0.05 \%$ sorbitan sesquiisostearate/hexane and sonicated for $4 \mathrm{~min}$. The suspensions prepared in TA and PC were diluted 100-fold with acetone and sonicated for $4 \mathrm{~min}$.

Scanning Electron Microscopic (SEM) Observation S-4300 (Hitachi High Technologies Corporation, Tokyo, Japan) was used for the SEM observation. The suspension was dropped on a multilayer wiping cloth (Kim towels ${ }^{\circledR}$, Cresia, Tokyo, Japan) and one piece from the top layer of the cloth was fixed to the sample stage. The sample was coated with palladium/platinum and observed under an acceleration voltage of $5.0 \mathrm{kV}$ and emission current of $20 \mu \mathrm{A}$.

Evaluation of the Dispersibility The dispersibilities of the riboflavin suspensions were determined by the method reported by Kitahara et al., with some modification. ${ }^{21)}$ One milliliter of each suspension was filled into screw glass vials (Tomsic Co., Ltd., Tokyo, Japan, capacity of $1.5 \mathrm{~mL}$; inner diameter of $12 \mathrm{~mm}$; height of $32 \mathrm{~mm}$ ), and the samples were stored at $25 \pm 2^{\circ} \mathrm{C}$ for 1 or 2 weeks. The dispersion ratio was expressed as the \% ratio of the height of the settling interface between the precipitate and the supernatant against the height of the entire system.

Rat Skin Preparation The permeation profiles of riboflavin through the rat skin were determined by in vitro permeation experiments. Male Sprague-Dawley (SD) rats (7 weeks old; Charles River Laboratories Japan, Inc., Atsugi, Kanagawa, Japan) were used for the skin permeation studies. The SD rats were euthanized by cervical dislocation under anesthesia with isoflurane, and the abdominal region of the SD rat was carefully shaved. One round section of the rat skin $(2.5 \mathrm{~cm}$ diameter) was excised from the abdominal skin. After subcutaneous fat was removed with scissors, the excised rat skin samples were frozen and stored at $-80^{\circ} \mathrm{C}$ until use (usually within 2 weeks). The care of the rats and the study protocols were in compliance with the "General Consideration for Animal Experiments," and were approved by the Ethics Committee for the Treatment of Laboratory Animals at Taisho Pharmaceutical Co., Ltd.

Skin Permeation Procedure The percutaneous absorption study was performed by a method outlined in Test Guideline 428 of the Organization for Economic Cooperation and Development. ${ }^{22)}$ The in vitro skin permeation experiments were mainly performed using modified Franz-type diffusion cells $\left(0.95 \mathrm{~cm}^{2}\right.$, diameter $\left.=1.1 \mathrm{~cm}\right){ }^{23)}$ Franz-type diffusion cells are usually used for determination of the permeation rates of drugs through the skin. The skin was thawed in a $\mathrm{CO}_{2}$ incubator (MCO-17AI; Sanyo Electric Co., Ltd., Osaka, Japan) at $32^{\circ} \mathrm{C}$ (relative humidity: $40-50 \%$ ) for $30 \mathrm{~min}$ before the start and was then mounted on diffusion cells. The receptor cell was filled with $3.1 \mathrm{~mL}$ of $0.1 \mathrm{M}$ phosphate buffer $(\mathrm{pH} 7.4)$. Ten milligram of the test suspensions was applied to the skin surface. The receptor cell medium was kept at $32^{\circ} \mathrm{C}$ and stirred with a Teflon stirrer driven at $150 \mathrm{rpm}$ by a constant speed motor throughout the experiment. The diffusion cells were maintained in a $\mathrm{CO}_{2}$ incubator at $32^{\circ} \mathrm{C}$ (relative humidity: 
40-50\%). The receptor fluid $(0.5 \mathrm{~mL})$ was sampled at $1,2,4$, 6,8 , and $24 \mathrm{~h}$, and the same volume of fresh $0.1 \mathrm{M}$ phosphate buffer ( $\mathrm{pH}$ 7.4) was added to the receptor cell to maintain a constant volume. The receptor fluid samples were stored in $4^{\circ} \mathrm{C}$ until the analysis of riboflavin concentrations.

Morphological Study To clarify the penetration of riboflavin into the skin, a morphological study was carried out using samples obtained after $24 \mathrm{~h}$ in the in vitro skin permeation experiments. The rat skin was cut out, embedded in $\mathrm{M}$. E. Cryo compound (Micro-edge Instruments Co., Ltd., Tokyo, Japan) using Cryomold ${ }^{\circledR}$ (Sakura Finetek Co., Ltd., Tokyo, Japan), and made frozen blocks were made in isopentane cooled with liquid nitrogen in order to prepare sections in the epidermis-dermis direction. Then, non-fixed frozen sections were prepared from these blocks using a cryostat (CM3050; Leica Microsystems K.K.), the samples were observed under a confocal laser scanning microscope (CLSM), TCS SP-5 (Leica Microsystems K.K.) using $488 \mathrm{~nm}$ argon laser (riboflavin: excitation maximum $445 \mathrm{~nm}$; emission maximum $530 \mathrm{~nm}$ ).

Sample Pretreatment and HPLC Conditions for Riboflavin The concentrations of riboflavin in the samples were determined using an HPLC system (LC-20AD system; Shimadzu Corporation, Kyoto, Japan) equipped with a fluorescence detector (RF-10AXL; Shimadzu Corporation). Briefly, $500 \mu \mathrm{L}$ of the sample solution was added to $500 \mu \mathrm{L}$ of organic solvent (acetonitrile-methanol $(1: 1, \mathrm{v} / \mathrm{v}))$, and mixed. After centrifugation at $15000 \mathrm{rpm}$ for $10 \mathrm{~min}$ at $5^{\circ} \mathrm{C}$, the supernatant $(20 \mu \mathrm{L})$ was directly injected into the HPLC system.

Chromatographic separation was performed using a YMC trait $(5 \mu \mathrm{m}, 4.6 \mathrm{~mm}$ i.d. $\times 150 \mathrm{~mm}$; YMC Co., Ltd., Tokyo, Japan) at $40^{\circ} \mathrm{C}$. The mobile phase was $0.01 \mathrm{M} \mathrm{NaH} \mathrm{PO}_{4}(\mathrm{pH}$ $5.5)-$ methanol $(65: 35, \mathrm{v} / \mathrm{v})$ at a flow rate of $0.8 \mathrm{~mL} / \mathrm{min}$. The wavelength of the fluorescence detector was set at $445 \mathrm{~nm}$ (excitation) and $530 \mathrm{~nm}$ (emission). The retention time was approximately $6 \mathrm{~min}$. The calibration curve range was from 5 to $500 \mathrm{ng} / \mathrm{mL}$ for riboflavin, and the accuracy and precision were calculated.

Statistical Analysis All data are shown as mean \pm S.D.
( $n=3$ or 4 ). The cumulative amounts of riboflavin permeated through the rat skin after $24 \mathrm{~h}$ topical application were compared between OC-NS and PD-NS, between OC-NS and CT-NS, and between PD-NS and CT-NS using the parametric Dunnett test. The lag times, permeation rates, and cumulative amounts of riboflavin permeated through the rat skin after $24 \mathrm{~h}$ topical application were compared between OC-NS and the suspension of intact riboflavin (OC-S) using Student's $t$-test. A value of $p<0.05$ was considered statistically signficiant. The statistical analysis was performed using the SAS software (ver. 9.2; SAS Institute Japan Ltd., Tokyo, Japan).

\section{Results and Discussion}

Physicochemical Properties of Non-aqueous Bases Table 1 shows the viscosity and solubility of riboflavin at $25^{\circ} \mathrm{C}$ in eight non-aqueous bases: LP, IM, PD, CT, OC, OL, TA, and PC. The viscosity of the bases ranged from 3.2 to $100 \mathrm{mPa} \cdot \mathrm{s}$. The density of TA and PC were 1.15 and $1.20 \mathrm{~g} / \mathrm{cm}^{3}$, higher than that of the other bases. Riboflavin, which is classified as a hydrophilic compound, scarcely dissolved in these eight bases. The solubility of riboflavin was the maximal in PC $(13 \mu \mathrm{g} / \mathrm{mL})$.

Table 1. Viscosity of the Non-aqueous Bases and Solubility of Riboflavin $\left(25^{\circ} \mathrm{C}\right)$

\begin{tabular}{cccc}
\hline $\begin{array}{c}\text { Non-aqueous } \\
\text { bases }\end{array}$ & $\begin{array}{c}\text { Viscosity } \\
(\mathrm{mPa} \cdot \mathrm{s})\end{array}$ & $\begin{array}{c}\text { Density } \\
\left(\mathrm{g} / \mathrm{cm}^{3}\right)\end{array}$ & $\begin{array}{c}\text { Solubility of } \\
\text { riboflavin }(\mu \mathrm{g} / \mathrm{mL})\end{array}$ \\
\hline LP & 100 & 0.86 & $-^{a)}$ \\
IM & 4.4 & 0.85 & $-^{a)}$ \\
PD & 7.9 & 0.92 & $-^{a)}$ \\
CT & 25 & 0.94 & $-{ }^{a)}$ \\
OC & 25 & 0.89 & 0.14 \\
OL & 27 & 0.84 & 2.1 \\
TA & 19 & 1.15 & 3.0 \\
PC & 3.2 & 1.20 & 13 \\
\hline
\end{tabular}

Each value represents the mean $(n=3)$. a) Less than the limit of quantitation $(0.048 \mu \mathrm{g} / \mathrm{mL})$.

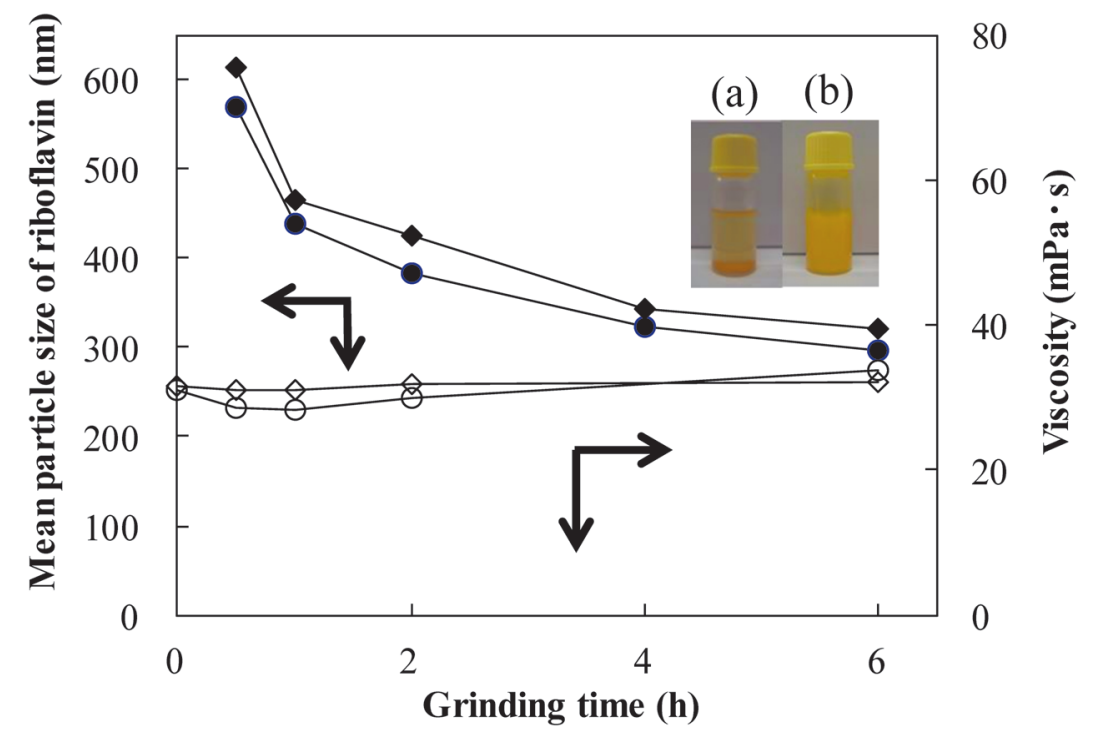

Fig. 1. Effect of Grinding Time and Bead Size on the Particle Size and Viscosity of Riboflavin Suspension in OC

Photographic images show samples with (a) intact and (b) ground with $0.5 \mathrm{~mm}$ beads for $6 \mathrm{~h}$. Key: ( $(\bullet)$ particle size; ground with $0.5 \mathrm{~mm}$ beads, ( $)$ particle size; ground with $1.0 \mathrm{~mm}$ beads, $(\bigcirc)$ viscosity; ground with $0.5 \mathrm{~mm}$ beads, $(\diamond)$ viscosity; ground with $1.0 \mathrm{~mm}$ beads. 


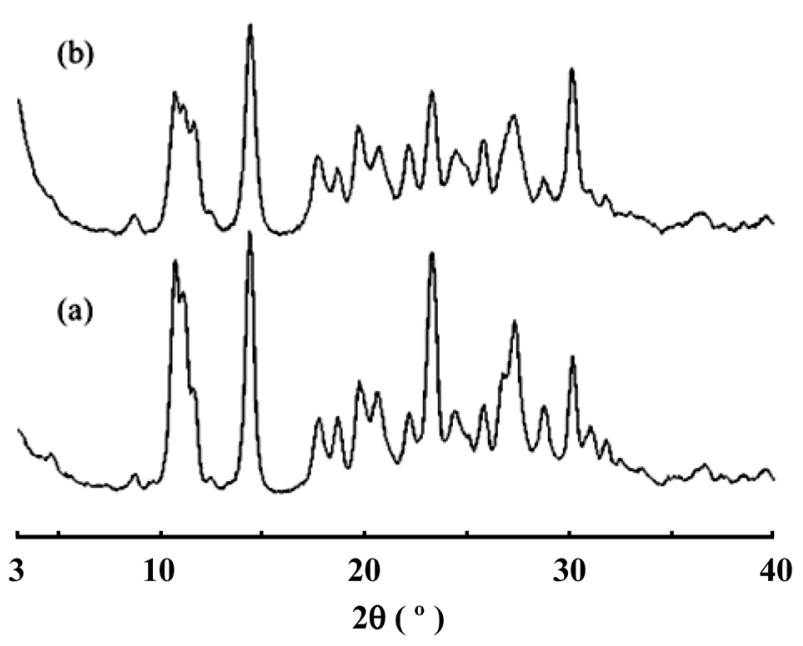

Fig. 2. Powder X-Ray Diffraction Patterns of (a) Intact Riboflavin and (b) Ground Riboflavin Separated from the OC-NS

The nanosuspension was prepared by grinding with $0.5 \mathrm{~mm}$ beads for $6 \mathrm{~h}$.

Evaluation of the Grinding Conditions for Riboflavin Bead Milling At first, we tried to determine the optimum grinding conditions in the preparation of riboflavin nanosuspension using $\mathrm{OC}$ as the dispersion medium. Figure 1 shows the effect of the grinding time and beads size on the particle size and on the viscosity of the riboflavin suspension. Grinding with $0.5 \mathrm{~mm}$ or $1.0 \mathrm{~mm}$ beads was associated with a decrease of the particle size with increasing grinding time. The particle size of riboflavin reached approximately $300 \mathrm{~nm}$ after grinding for $6 \mathrm{~h}$. No significant change in the viscosity was observed during the grinding. In addition, the temperature was kept at approximately $30^{\circ} \mathrm{C}$ without artificial control at room temperature.

We evaluated the grinding performance using small-sized zirconia beads $(0.1 \mathrm{~mm}$ diameter $)$ in the same manner shown in Fig. 1. However, no reduction to submicron particle size was observed after $6 \mathrm{~h}$ of grinding.

The crystallinity of riboflavin in the nanosuspensions was evaluated after grinding. The particles of nanonized riboflavin were separated from the nanosuspension prepared by grinding with $0.5 \mathrm{~mm}$ beads in $\mathrm{OC}$ for $6 \mathrm{~h}$. The powder X-ray diffraction patterns of intact and nanonized riboflavin are shown in Fig. 2. The results indicated that amorphization of riboflavin was scarcely observed during grinding in non-aqueous base. It was shown that the nanosuspension prepared by grinding in non-aqueous bases had the advantage of maintained physical stability. As an optimum grinding condition, a bead size of $0.5 \mathrm{~mm}$ and grinding time of $6 \mathrm{~h}$ were chosen for the subsequent investigations.

Evaluation of the Particle Size and Dispersibility Nanosuspensions were prepared by grinding with $0.5 \mathrm{~mm}$ beads for $6 \mathrm{~h}$. Table 2 shows the mean particle size of riboflavin in the nanosuspensions. The particle size ranged from 206 to $469 \mathrm{~nm}$. An SEM image of OC-NS is presented in Fig. 3. The particles appeared to be agglomerates with a smooth surface. The particle size of riboflavin appeared to be less than $500 \mathrm{~nm}$, which was consistent with that determined by the dynamic light scattering method. The particle morphology of riboflavin in the other non-aqueous bases was similar to that observed in the SEM image shown in Fig. 3. Bead milling could be used to prepare riboflavin nanosuspension in all the
Table 2. Particle Size of Riboflavin and Dispersion Ratio of Riboflavin Nanosuspensions Prepared by Bead Milling for $6 \mathrm{~h}$ Storage Condition Is at $25 \pm 2{ }^{\circ} \mathrm{C}$ for 1 Week and 2 Weeks

\begin{tabular}{cccc}
\hline \hline & & \multicolumn{2}{c}{ Dispersion ratio (\%) } \\
\cline { 3 - 4 } Sample name & $\begin{array}{c}\text { Mean particle size } \\
(\mathrm{nm})\end{array}$ & $\begin{array}{c}\text { Storage for } \\
1 \text { week }\end{array}$ & $\begin{array}{c}\text { Storage for } \\
2 \text { weeks }\end{array}$ \\
\hline OL-NS & $206 \pm 7$ & $86 \pm 2$ & $74 \pm 1$ \\
PC-NS & $217 \pm 21$ & $94 \pm 1$ & $88 \pm 0$ \\
TA-NS & $219 \pm 7$ & $99 \pm 1$ & $98 \pm 0$ \\
OC-NS & $345 \pm 17$ & $91 \pm 2$ & $76 \pm 1$ \\
IM-NS & $347 \pm 45$ & $60 \pm 2$ & $55 \pm 2$ \\
CT-NS & $366 \pm 12$ & $94 \pm 2$ & $85 \pm 1$ \\
PD-NS & $420 \pm 8$ & $79 \pm 4$ & $77 \pm 4$ \\
LP-NS & $469 \pm 32$ & $40 \pm 1$ & $35 \pm 0$ \\
\hline
\end{tabular}

Each value represents the mean \pm S.D. $(n=3)$.

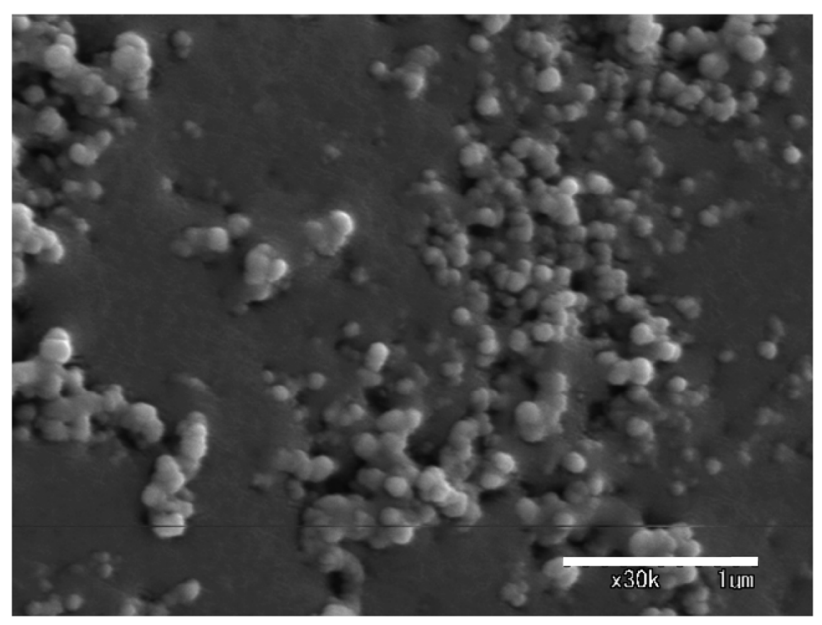

Fig. 3. SEM Image of OC-NS Prepared by Grinding with $0.5 \mathrm{~mm}$ Beads for $6 \mathrm{~h}$

The scale bar indicates $1 \mu \mathrm{m}$.

non-aqueous bases used.

Table 2 also represents the dispersion ratio of riboflavin nanosuspension after storage at $25^{\circ} \mathrm{C}$ for 1 and 2 weeks. Riboflavin, which is classified as a hydrophilic compound, was well dispersed in all six of the non-aqueous bases other than LP and IM. The dispersion ratio after storage for 2 weeks varied depending on the bases, from 35 to $98 \%$. Stokes' law ${ }^{24)}$ states that

$$
W_{\mathrm{s}}=D_{\mathrm{s}}^{2} g\left(\rho_{\mathrm{s}}-\rho\right) / 18 \mu,
$$

where $W_{\mathrm{s}}, D_{\mathrm{s}}$ and $\rho_{\mathrm{s}}$ are the settling velocity, diameter and density of the particle, respectively, $\rho$ and $\mu$ are the density and viscosity of the dispersion medium, respectively, and $g$ is the gravitational acceleration. The particle dispersion became more and more stable with increasing viscosity of the bases and reducing density difference between the particle and base (Table 1). The riboflavin density was calculated as $1.65 \mathrm{~g} / \mathrm{cm}^{3}$ by ACD/Percepta Platform (ver. 2012, Advanced Chemistry Development Inc., Toronto, Canada). As expected, the nanosuspension of TA with a relatively higher viscosity and density in these bases showed the greatest dispersibility. However, the nanosuspension of PC showed the second highest dispersibility despite showing the lowest viscosity. We speculated 


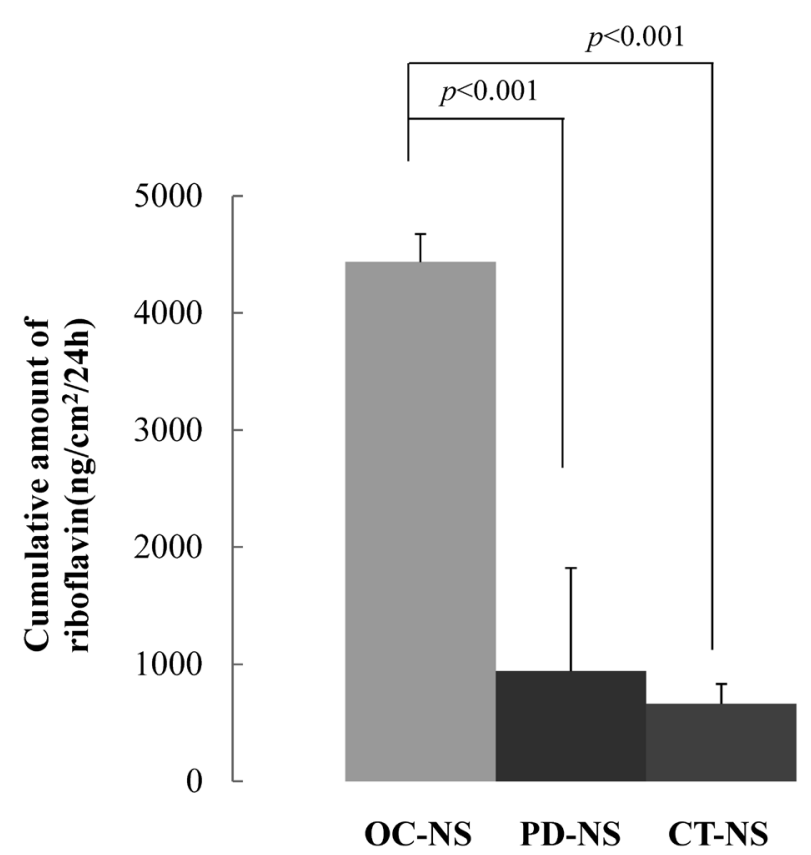

Fig. 4. Cumulative Amounts of Riboflavin Permeated through the Rat Skin after 24h Topical Application of OC-NS, PD-NS, and CT-NS, Respectively

Each value represents the mean + S.D. $(n=3), p<0.001$ was observed between OC-NS and PD-NS, and between OC-NS and CT-NS, no significiant difference $(p<0.05)$ was observed between PD-NS and CT-NS using parametric Dunnett tests.

that the density of the base had a significant impact on the dispersibility of the nanoparticles. Furthermore, PC is known as the polar aprotic base having a high dielectric constant, ${ }^{25}$ ) unlike oily bases (nonpolar aprotic base). We considered that the magnitude of the dielectric constant of PC contributed to its high dispersibility. Among the oily bases IM, PD, CT, OC, and OL, the dispersion ratio of CT-NS was the best, because of its higher viscosity and density, while the dispersion ratio of IM with its lower viscosity and density was not as good. On the other hand, the nanosuspension of LP showed the lowest dispersion ratio. This phenomenon could not be explained based on the viscosity or density. $\mathrm{Wu}$ et al. reported that the dispersibility of cobalt nanoparticles in hexane could be enhanced by OC chemisorbed onto the cobalt surface. ${ }^{26)} \mathrm{LP}$ consists of saturated hydrocarbons without functional groups and has lower affinity for riboflavin than the other bases. It was considered that riboflavin suspended in LP could easily become agglomerated and settle out because of the poor compatibility. For stabilization of the particle dispersion in nonaqueous bases and improving the dispersibility of nanoparticles, the affinity for riboflavin as well as the viscosity and density of non-aqueous bases is considered to be important.

In addition, the dispersion ratios after 1 week of storage of CT-NS, OC-NS, and OL-NS were higher as compared to those of IM-NS or PD-NS. Thus, it is thought that a high-viscosity base has the ability to maintain a well-dispersed state for a short time.

Optimum Dispersion Medium for Riboflavin Suspension Among the eight non-aqueous bases used, OC, PD, and CT were selected as the optimal dispersion media for riboflavin, due to the low solubility and high dispersion ratio of riboflavin in these media. Figure 4 shows the cumulative amounts of riboflavin permeated through the rat skin after $24 \mathrm{~h}$ topical
Table 3. Lag Times, Permeation Rates, and Cumulative Amounts of Riboflavin Permeated through the Rat Skin after Topical Application of OC-NS or OC-S

\begin{tabular}{lccc}
\hline \hline Sample name & $\begin{array}{c}\text { Lag time } \\
(\mathrm{h})\end{array}$ & $\begin{array}{c}\text { Permeation rate } \\
\left(\mathrm{ng} / \mathrm{cm}^{2} / \mathrm{h}\right)\end{array}$ & $\begin{array}{c}\text { Cumulative amount } \\
\left(\mathrm{ng} / \mathrm{cm}^{2}\right)\end{array}$ \\
\hline OC-S & $0.0 \pm 0.1$ & $30.3 \pm 15.0$ & $843 \pm 335$ \\
OC-NS & $0.3 \pm 0.4$ & $89.1 \pm 23.9 * *$ & $2345 \pm 813^{*}$ \\
\hline
\end{tabular}

Each value represents the mean \pm S.D. $(n=4)$. Stastical analysis was performed by Student's $t$-test. *: Significiant at $p<0.05$ compared with OC-S, **: Significiant at $p<0.01$ compared with OC-S.

application of OC-NS, PD-NS, and CT-NS. The permeated amount of OC-NS was 3-6 times greater than that of PD-NS or CT-NS. OC is well known as one of the enhancers of skin permeation by increasing the fluidity of the lipid in the stratum corneum. ${ }^{27,28)}$ The nature of OC could contribute to the penetration of the nanoparticles into the stratum corneum. Therefore, OC was selected as the dispersion medium for the riboflavin nanosuspension in the subsequent experiments.

Evaluation of the Skin Permeation of Riboflavin from Nanosuspension The skin permeability of OC-NS was investigated in comparison with that of OC-S. Table 3 shows the cumulative amounts of riboflavin that had permeated through the rat skin after topical application of OC-S or OC-NS. The cumulative riboflavin amounts increased linearly in both cases, and the values at $24 \mathrm{~h}$ after topical application of OC-S or OC-NS reached 843 and $2345 \mathrm{ng} / \mathrm{cm}^{2}$, respectively. The cumulative amounts and permeation rates of OC-NS were apporoximately three times greater than those observed in OC-S. This result suggests that nanonization of riboflavin markedly improved its skin permeability. Many researchers have reported that nanoparticles penetrate into the hair follicles. ${ }^{29-31)}$ Todo et al. reported that polystyrene latex nanoparticles (mean particle size $500 \mathrm{~nm}$ ) penetrated into the hair follicles of porcine skin. ${ }^{32)}$ On the other hand, Tahara et al. reported that a $\mathrm{S} / \mathrm{O}$ nanosuspension of protein (mean particle size: $214-257 \mathrm{~nm}$ ) penetrated into the stratum corneum in the in vitro Yucatan micropig skin permeation experiments. ${ }^{11)}$

Thus, skin permeation enhancement of riboflavin nanosuspension using OC could be achieved not only into the hair follicles, but also into the stratum corneum.

Next, a morphological study was performed to clarify the penetration of riboflavin into the hair follicles and/or stratum corneum. Figure 5 represents the CLSM images at $24 \mathrm{~h}$ after topical application of OC-S or OC-NS in the in vitro skin permeation experiments. The riboflavin-derived autofluorescence intensity of the epidermis after application of OC-NS was stronger than that after application of OC-S. However, the difference of the intensity in the hair follicles between the two suspensions was not as clear as that in the epidermis in this study, and it is possible that the nanoparticles penetrate into the hair follicles from previous reports. ${ }^{8-10)}$ For these reasons, we suggest that nanonization of riboflavin using OC could improve its penetration into the stratum corneum.

From the results, it is concluded that bead milling in a non-aqueous base is one of the simple methods to prepare nanosuspensions of hydrophilic drugs such as riboflavin and improve their skin permeability.

Study on Stabilization of the Nanosuspensions in Oleic Acid A further study on the improvement of the dispersibility using nonionic surfactants was conducted in view of the 

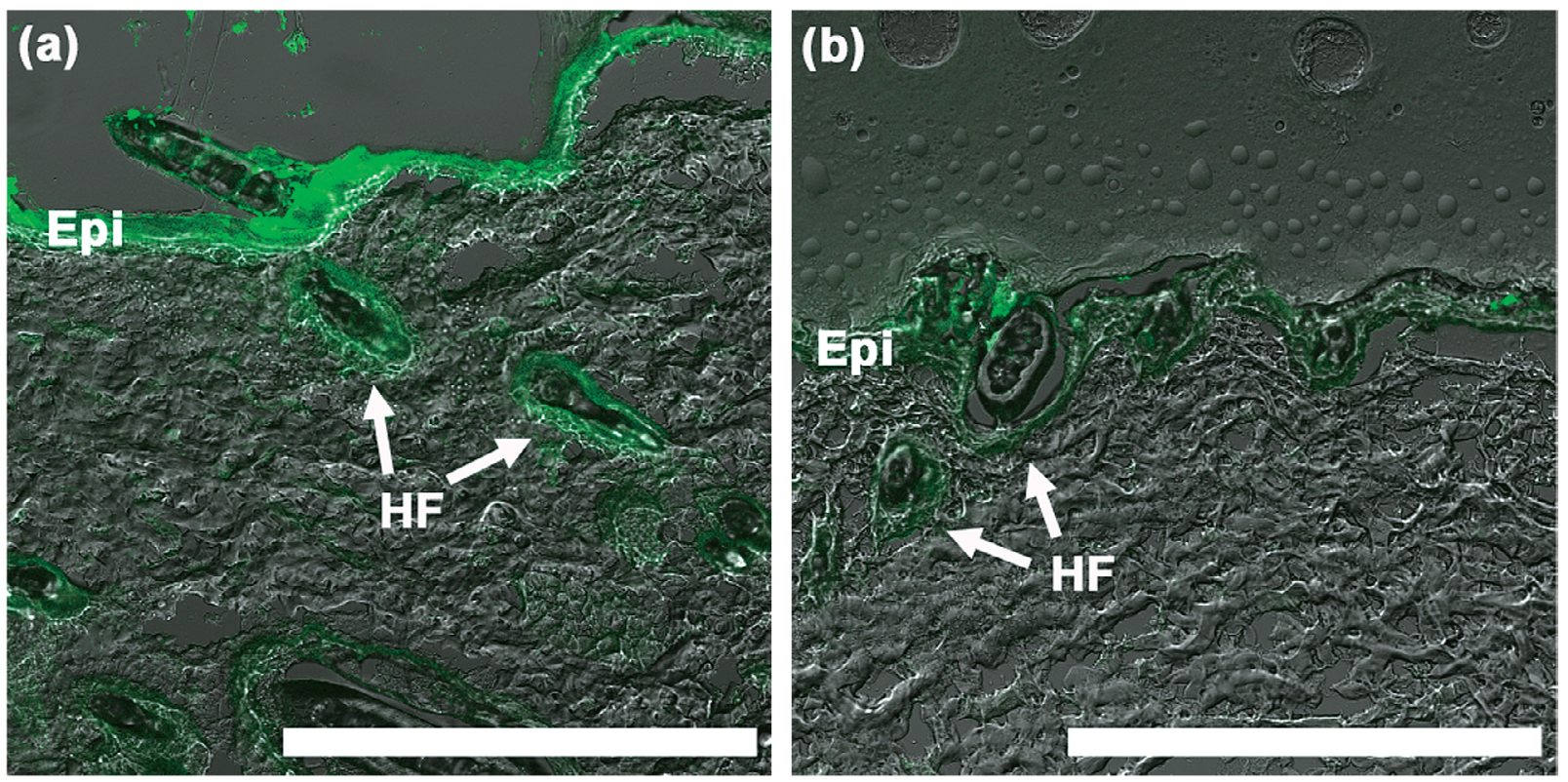

Fig. 5. CLSM Images of the Rat Skin Cross-Sections after in Vitro Skin Permeation Experiment of (a) OC-NS and (b) OC-S Using Franz-Type Diffusion Cells

Scale bar, Epi, and HF represent $250 \mu \mathrm{m}$, epidermis, and hair follicle, respectively.

Table 4. Dispersion Ratio of Riboflavin Nanosuspensions with and without Addition of Surfactant (ST, SE, PS, and PP) after the Storage at $25 \pm 2{ }^{\circ} \mathrm{C}$ for 1 Week and 2 Weeks

\begin{tabular}{lcc}
\hline \hline \multirow{2}{*}{ Sample name } & \multicolumn{2}{c}{ Dispersion ratio (\%) } \\
\cline { 2 - 3 } & Storage for 1 week & Storage for 2 weeks \\
\hline OC (OC-NS) & $91 \pm 2$ & $76 \pm 1$ \\
OC/ST & $85 \pm 2$ & $73 \pm 2$ \\
OC/SE & $92 \pm 1$ & $83 \pm 1$ \\
OC/PS & $100 \pm 0$ & $99 \pm 1$ \\
OC/PP & $100 \pm 0$ & $99 \pm 1$ \\
\hline
\end{tabular}

Each value represents the mean \pm S.D. $(n=3)$.

application to a dosage form of the nanosuspension. The nanosuspensions containing surfactant were prepared by the addition of $5.0 \%$ sorbitan trioleate $(\mathrm{OC} / \mathrm{ST})$, sucrose erucate $(\mathrm{OC} /$ $\mathrm{SE})$, polysorbate 65 (OC/PS), or polyglyceryl-6 polyricinoleate (OC/PP) to OC-NS, respectively. Table 4 represents the dispersion ratios of the riboflavin nanosuspensions after storage at $25^{\circ} \mathrm{C}$ for 1 and 2 weeks. Addition of ST led to a decrease in the dispersibility of OC-NS. Addition of SE could not enhance the dispersion ratio in comparison to that in OC. On the other hand, addition of PS and PP significantly improved the dispersibility, and precipitation was hardly observed even after 2 weeks of storage. Affinity between riboflavin and the base is important to obtain stable dispersion of the nanosuspension, as mentioned above. In addition, it is reported that steric repulsion generated by the substance adsorbed on to the particle surface is effective for improving the dispersion of a suspension. ${ }^{33)}$ Sorbitan trioleate, SE, PS, and PP are esters mainly composed of OC (C18), erucic acid (C22), stearic acid (C18), and ricinoleic acid (C18), respectively. Sorbitan trioleate has a structure in which OC is ester-linked to sorbitol. The deteriorated dispersibility observed by the addition of ST could be explained as being because the amount of $\mathrm{OC}$ adsorbed on to the riboflavin surface would be reduced as compared to that of $\mathrm{OC}$ due to the steric hindrance by the sorbitol moiety interacting with the riboflavin surface. The difference in the dispersibility between addition of SE and ST could be explained in terms of the length of the side chains which could affect the dispersibility. However, PS has a structure similar to that of ST, and the side chains are lengthened by the addition of ethylene oxide. Polyglyceryl-6 polyricinoleate has long side chains, so that some ricinoleic acid moieties are bonded to each other. We considered that the improved dispersibility of the nanosuspensions arising from the addition of PP or PS, which have long side chains, was due to steric repulsion.

From the results, it is suggested that the technology using bead milling could be applicable for hydrophilic drugs with low $\log K_{\mathrm{o} / \mathrm{w}}$ by optimizing the dispersion medium and surfactant.

The cumulative amounts of riboflavin permeated through the rat abdominal skin observed for riboflavin nanosuspensions in OC/PS and OC/PP were compared with that for the suspension in OC (without addition of surfactant). The ratios of the riboflavin cumulative amounts and permeation rates of nanosuspensions in OC, OC/PS and OC/PP were $1: 0.90: 0.97$ and $1: 0.94: 1.02$. While the addition of a surfactant to the nanosuspension could improve the riboflavin dispersibility, no significant differences in the permeation amount and rate was observed among the three nanosuspensions. It was thought that OC-NS had a sufficient number of nanoparticles that could penetrate into the hair follicles or the stratum corneum.

\section{Conclusion}

Non-aqueous nanosuspensions of riboflavin were prepared by grinding riboflavin with zirconia beads using eight nonaqueous bases. The mean particle size of the riboflavin in the prepared suspensions ranged from 206 to $469 \mathrm{~nm}$, as determined by the dynamic light scattering method. Among these suspensions, the riboflavin suspension in OC as the dispersion medium was selected for determination of the dispersibility 
and skin permeability. The cumulative amount and permeation rate of riboflavin from the nanosuspension prepared in $\mathrm{OC}$ were approximately three times higher than those for unprocessed riboflavin in OC. Furthermore, addition of polysorbate 65 or polyglyceryl-6 polyricinoleate to OC-NS markedly improved the riboflavin dispersibility. Since preparation of drug nanosuspension by bead milling is a simple and easy method, it is applicable to develop a formulation to improve the skin permeability of hydrophilic drugs by selecting appropriate dispersion medium and surfactant.

Acknowledgment We would like to thank Dr. Akio Miwa and Mr. Tsuyoshi Endo (Taisho Pharmaceutical Co., Ltd.) for their excellent technical support.

Conflict of Interest Takayuki Sato, Hiroyuki Takeuchi, Takanobu Sakurai, Kensuke Tanaka, and Kota Matsuki are employees of Taisho Pharmaceutical Co., Ltd.

\section{References}

1) Morimoto Y., Hatanaka T., Sugibayashi K., Omiya H., J. Pharm. Pharmacol., 44, 634-639 (1992).

2) Flynn G. L., Stewart B., Drug Dev. Res., 13, 169-185 (1988).

3) Desai P., Patlolla R. R., Singh M., Mol. Membr. Biol., 27, 247-259 (2010).

4) Liu K. C., Green C. R., Alany R. G., Rupenthal I. D., Int. J. Pharm., 441, 687-692 (2013).

5) Gratieri T., Kalaria D., Kalia Y. N., Expert Opin. Drug Deliv., 8, 645-663 (2011).

6) Matriano J. A., Cormier M., Johnson J., Young W. A., Buttery M., Nyam K., Daddona P. E., Pharm. Res., 19, 63-70 (2002).

7) Cormier M., Johnson B., Ameri M., Nyam K., Libiran L., Zhang D. D., Daddona P., J. Control. Release, 97, 503-511 (2004).

8) Tsujimoto H., Hara K., Tsukada Y., Huang C. C., Kawashima Y., Arakaki M., Okayasu H., Mimura H., Miwa N., Bioorg. Med. Chem. Lett., 17, 4771-4777 (2007).

9) Raber A. S., Mittal A., Schäfer J., Bakowsky U., Reichrath J., Vogt T., Schaefer U. F., Hansen S., Lehr C. M., J. Control. Release, 179, 25-32 (2014).

10) Hasanovic A., Zehl M., Reznicek G., Valenta C., J. Pharm. Pharmacol., 61, 1609-1616 (2009).

11) Tahara Y., Honda S., Kamiya N., Piao H., Hirata A., Hayakawa E., Fujii T., Goto M., J. Control. Release, 131, 14-18 (2008).
12) Kohli A. K., Alpar H. O., Int. J. Pharm., 275, 13-17 (2004).

13) Müller R. H., Radtke M., Wissing S. A., Adv. Drug Deliv. Rev., 54 (Suppl 1), S131-S155 (2002).

14) Domínguez-Delgado C. L., Rodríguez-Cruz I. M., Escobar-Chávez J. J., Calderón-Lojero I. O., Quintanar-Guerrero D., Ganem A., Eur. J. Pharm. Biopharm., 79, 102-107 (2011).

15) Mitri K., Shegokar R., Gohla S., Anselmi C., Müller R. H., Int. J. Pharm., 414, 267-275 (2011).

16) Kondo N., Iwao T., Masuda H., Yamanouchi K., Ishihara Y., Yamada N., Haga T., Ogawa Y., Yokoyama K., Chem. Pharm. Bull., 41, 737-740 (1993).

17) Takatsuka T., Endo T., Jianguo Y., Yuminoki K., Hashimoto N., Chem. Pharm. Bull., 57, 1061-1067 (2009).

18) Niwa T., Miura S., Danjo K., Int. J. Pharm., 405, 218-227 (2011).

19) National Institute for Occupational Safety and Health, Centers for Disease Control and Prevention: Atlanta, GA, 2001. "RIBOFLAVIN, International Chemical Safety Cards No. 1454 (U.S. National Version).”: 〈http://www.cdc.gov/niosh/ipcsneng/neng1454.html〉 cited March, 2014.

20) Filipowicz A., Wołowiec S., Int. J. Pharm., 408, 152-156 (2011).

21) Kitahara A., Tamura T., Matsumura S., Chem. Lett., 8, 1127-1128 (1979).

22) "OECD, Test Guideline 428. Skin absorption: in vitro method," OECD, Paris (2004).

23) Franz T. J., J. Invest. Dermatol., 64, 190-195 (1975).

24) Le Roux J. P., Sediment. Geol., 178, 285-313 (2005).

25) Payne R., Theodorou I. E., J. Phys. Chem., 76, 2892-2900 (1972).

26) Wu N., Fu L., Su M., Aslam M., Wong K. C., Dravid V. P., Nano Lett., 4, 383-386 (2004).

27) Naik A., Pechtold L. A. R. M., Potts R. O., Guy R. H., J. Control. Release, 37, 299-306 (1995).

28) Rowat A. C., Kitson N., Thewalt J. L., Int. J. Pharm., 307, 225-231 (2006).

29) Lademann J., Richter H., Teichmann A., Otberg N., Blume-Peytavi U., Luengo J., Weiß B., Schaefer U. F., Lehr C. M., Wepf R., Sterry W., Eur. J. Pharm. Biopharm., 66, 159-164 (2007).

30) Shim J., Seok Kang H., Park W. S., Han S. H., Kim J., Chang I. S., J. Control. Release, 97, 477-484 (2004).

31) Patzelt A., Lademann J., Expert Opin. Drug Deliv., 10, 787-797 (2013).

32) Todo H., Kimura E., Yasuno H., Tokudome Y., Hashimoto F., Ikarashi Y., Sugibayashi K., Biol. Pharm. Bull., 33, 1394-1399 (2010).

33) Hadziioannou G., Patel S., Granick S., Tirrell M., J. Am. Chem. Soc., 108, 2869-2876 (1986). 